\title{
Benefícios da combinação do treinamento manipulativo osteopático, exercícios aquáticos e ginástica respiratória para a qualidade de vida em crianças e adolescentes asmáticos
}

\begin{tabular}{|c|c|}
\hline $\begin{array}{l}\text { Sara Teresinha CORAZZA* } \\
\text { Felipe de Oliveira SCHERER }{ }^{* * *} \\
\text { Silvana Corrêa MATHEUS* } \\
\text { Rafael Cunha LAUX***** }\end{array}$ & $\begin{array}{l}\text { *Centro de Educação } \\
\text { Física e Desporto, } \\
\text { Universidade Federal } \\
\text { de Santa Maria, Santa } \\
\text { Maria, RS, Brasil. } \\
{ }^{* *} \text { Universidade } \\
\text { Federal de Santa } \\
\text { Maria, Santa Maria, } \\
\text { RS, Brasil. } \\
\text { ***Universidade } \\
\text { do Oeste de Santa } \\
\text { Catarina, Chapecó, } \\
\text { SC, Brasil. }\end{array}$ \\
\hline
\end{tabular}

\section{Resumo}

Desenvolver programas de exercício físico para a melhoria da qualidade de vida em asmáticos podem influenciar positivamente nas realizações de atividades sociais e esportivas por esse público. 0 objetivo deste estudo, foi investigar o efeito da adição do Tratamento Manipulativo Osteopático (TMO) às sessões de exercícios de ginástica respiratória e exercícios aquáticos na melhoria da qualidade de vida de asmáticos. 0 estudo foi desenvolvido com 18 participantes, com idade entre 8 e 15 anos, de ambos os sexos e estudantes do Ensino Fundamental I e II. Onze (11) fizeram parte do grupo experimental 1(GE1) e realizaram o programa combinado de TMO, atividades aquáticas e a ginástica respiratória. Sete (7) sujeitos fizeram parte do grupo experimental 2 (GE2) que realizaram somente atividades aquáticas e a ginástica respiratória. Ambos foram testados antes e após a realização de 16 sessões de TMO e 32 sessões das demais práticas, através do Questionário de Qualidade de Vida para Asma Pediátrica (PedsQL). Para análise dos dados, após comprovação da normalidade, utilizou-se o teste T de Student para amostras pareadas e o teste T para amostras independentes, através do aplicativo SPSS, versão 21.0 com nível de significância de 5\%. Os resultados obtidos comprovaram a diferença estatisticamente significativa nas dimensões física, social, escolar e emocional do questionário no GE, quando comparados entre o início e o final do programa e confirmaram a diferença estatisticamente significativa quando comparados os pós testes entre os grupos, prevalecendo as melhorias em todas dimensões para 0 grupo que realizou a TMO como diferencial. Os resultados fortalecem a ideia da eficiência da conjugação dessas práticas na melhoraria da qualidade de vida das crianças asmáticas leves e moderadas.

PalAVRAS-Chave: Asma; Terapia por Exercícios; Manipulação Osteopática; Natação; Exercícios

Respiratórios.

\section{Introdução}

A asma é uma doença inflamatória crônica das vias aéreas que causa obstrução no fluxo do ar e traz muitos prejuízos aos seus portadores. Ela pode ser desencadeada por agentes alérgicos, irritantes, alteraçóes de clima e até por exercício mal orientado ${ }^{1,2}$. A prevalência da asma vem aumentando nas últimas décadas, sendo considerada, atualmente, uma das principais doenças crônicas no mundo. Dados da Organização Mundial da Saúde (OMS) estimam que até o final de 2025 serão mais de 100 milhóes de pacientes asmáticos no mundo 3 . A presença dessa patologia implica modificaçóes em algumas dimensóes da vida do indivíduo podendo afetar o sono, os hábitos alimentares, aspectos emocionais e a qualidade de vida ${ }^{4-6}$.

Muitos estudos comprovaram que entre os benefícios da atividade física para pacientes asmáticos 
está a redução da frequência de ocorrência de crises de asma e promoção de uma boa ventilação pulmonar, podendo estes exercícios serem indicados, desde que combinados ao uso de medicamentos ${ }^{2,5,7-12}$. Ainda, as atividades físicas são provedoras de ganhos na mecânica respiratória, na prevenção de desordens posturais, melhorando a condição física geral dos indivíduos asmáticos e contribuindo para o desenvolvimento das aptidóes físicas e motoras ${ }^{13}$ $\mathrm{e}$, consequentemente, para a qualidade de vida ${ }^{14}$.

É conveniente destacar que existem exercícios considerados provocadores de crises, entre eles a corrida, a caminhada forte, pedalar uma bicicleta, e também os menos provocadores como a natação, desde que executada lentamente com ênfase na mecânica expiratória. A natação, portanto, é muito recomendada aos asmáticos por desenvolver-se num ambiente úmido e adequado ao bom funcionamento da função respiratória, ventilação pulmonar, fortalecimento da musculatura respiratória, corporal geral e prevençáo de alteraçōes na coluna vertebral ${ }^{15,16}$. Outro exercício que tem sido apontado como benéfico para a população asmática é a ginástica respiratória ${ }^{17} \mathrm{com}$ o foco no relaxamento de Jacobson ${ }^{18,19}$, fortalecimento muscular, desbloqueio torácico e respiração diafragmática ${ }^{12,20}$.

A literatura exibe estudos utilizando o tratamento manipulativo osteopático (TMO) beneficiando indivíduos com desordens respiratórias ${ }^{21,22}$, destacando algumas pesquisas em que esse tratamento mostrou-se eficaz quando aplicados em crianças asmáticas ${ }^{21,23}$ jovens e adultos ${ }^{24}$. A osteopatia foi fundamentada pelo Médico Andrew Taylor Still em meados de 1874 nos Estados Unidos da América, tratando-se de uma técnica que utiliza o tratamento manual e a sensibilidade das máos, através de manobras buscando disfunções de partes do corpo humano, visando a homeostase. OTMO é um tratamento de baixo custo, que traz ao paciente a sensação de melhora e que auxilia na diminuição da morbidade e mortalidade, fatores esses associados frequentemente à população pediátrica com asma ${ }^{23}$.

A identificação da utilização da TMO foi alvo de pesquisa em 2003, em Ohio - EUA ${ }^{25}$, e os resultados foram preocupantes, pois de 871 médicos que responderam o questionário online, aproximadamente $75 \%$ nunca utilizavam ou raramente utilizavam TMO, $44 \%$ dos que responderam o questionário não utilizavam TMO e $31 \%$ responderam que tratavam menos de 10 pacientes com TMO por semana, antes da pesquisa. No entanto, desde 2003 aos dias atuais percebeu-se uma mudança em relação ao uso ${ }^{26-31}$, projetando-se em diferentes patologias, inclusive na asma.

Baseando-se nos argumentos anteriores sobre os efeitos benéficos de diferentes atividades com asmáticos, justifica-se a realização deste estudo, a fim de se conhecer os efeitos de um programa inovador na literatura, conveniente para essa população asmática e faixa etária, priorizando o baixo custo e conjugando exercícios físicos em meio líquido e terapia manual.

Portanto, o objetivo do estudo foi investigar o efeito da adiçáo do Tratamento Manipulativo Osteopático (TMO) às sessões de exercícios de ginástica respiratória e exercícios aquáticos na melhoria da qualidade de vida de asmáticos.

\section{Método}

O presente estudo, foi analisado e aprovado pelo Comitê de Ética da UFSM com CAAE $\mathrm{n}^{\circ} 27035814.0 .0000 .5346$. Iniciou-se os procedimentos após a assinatura do Termo de Consentimento Livre e Esclarecido (TCLE) pelos pais dos alunos.

O estudo, caracterizado por experimental ${ }^{32}$, começou com 24 participantes, com idade entre 8 e 15 anos. Adotou-se como critérios de inclusão ser asmático e estar matriculado no Projeto de Extensão Natação e Ginástica Respiratória para Crianças e Adolescentes Asmáticos (registro no GAP no 336331), desenvolvido no Conjunto de Piscina Térmica do Centro de Educação Física e Desportos (CEFD) da Universidade Federal de Santa Maria (UFSM). Todos foram diagnosticados com asma leve ou moderada através dos critérios do questionário de classificação da asma ${ }^{33}$. Os alunos foram divididos através de um sorteio simples randomizado, em dois grupos de 12 sujeitos, GE1 e o GE2. Na realização das atividades houve a exclusão de seis (6) sujeitos no estudo, em funçáo das ausências nas intervenções, ocasionadas pelas baixas temperaturas e chuvas constantes, ficando desta forma 18 participantes, 11 no GE1 e 7 no GE2. Foram adotados como critérios de exclusão não assinar o TCLE, não responder ao questionário e ter ausência superior a $25 \%$ do total de aulas.

A TABELA 1 representa os dois Grupos (GE1 e GE2), o a idade, massa corporal e estatura. 
TABELA 1 - Caracterização da amostra

\begin{tabular}{lcc}
\hline Variáveis & Grupo experimental 1 & Grupo experimental 2 \\
& Média \pm DP & Média \pm DP \\
\hline Idade (anos) & $11,53 \pm 2,31$ & $12,20 \pm 2,92$ \\
Massa $(\mathrm{kg})$ & $44,02 \pm 10,47$ & $45,12 \pm 13,19$ \\
Estatura $(\mathrm{cm})$ & $1,37 \pm 0,12$ & $1,40 \pm 0,15$ \\
\hline
\end{tabular}

\section{Instrumento de Qualidade de Vida}

Como instrumento avaliativo utilizou-se Questionário de Qualidade de Vida para Asma Pediátrica (PedsQL $\left.{ }^{\mathrm{TM}}\right)^{34,35}$ validado para o idioma português. Este instrumento serviu para avaliar os efeitos da combinação do tratamento manipulativo osteopático (TMO), exercícios aquáticos e ginástica respiratória.

O instrumento avalia a qualidade de vida relacionada à saúde em crianças e adolescentes entre 2 e 18 anos e é composto por 23 questóes divididas em 4 dimensões: funcionamento físico (8 itens), funcionamento emocional ( 5 itens), funcionamento social (5 itens) e funcionamento escolar (5 itens). As questóes referem-se à duração de um problema no último mês, sendo pontuados de 0 (nunca é problema), 1 (quase nunca é problema), 2 (algumas vezes é problema), 3 (muitas vezes é problema) a 4 (quase sempre é problema). Deste modo, pontuações mais baixas revelam melhor qualidade de vida. Por fim, somando-se os itens de cada dimensão e dividindo- se pelo número de itens que cada uma contém, obtém-se o valor de qualidade de vida específica de cada dimensáo. Este instrumento de avaliaçáo foi preenchido pela criança/adolescente, enfatizando a sua própria percepçáo da qualidade de vida, mas explicado pelo avaliador quando necessário.

\section{Delineamento do estudo}

A intervenção foi realizada num período de 16 semanas num total de 32 sessóes com todas as atividades, com exceçáo do TMO que foi realizado em 16 sessóes - uma vez na semana. Justifica-se a aplicação somente em uma sessão semanal (em $50 \%$ das aulas), considerando que a osteopatia é uma técnica que preconiza em sua aplicação o intervalo de 3 a 5 dias para que o equilíbrio corpóreo aconteça ${ }^{36}$. No dia que náo acontecia a TMO aumentava-se o tempo da técnica de exercícios aquáticos. O tempo total na realização foi de 55 min de duração em cada sessão. Ela foi desenvolvida, conforme FIGURA 1. Estrutura representativa do estudo:

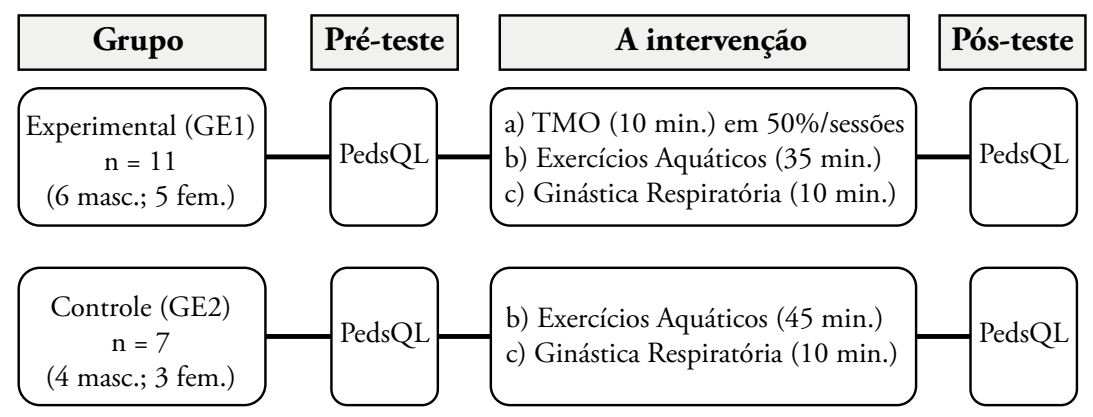

FIGURA 1 - Apresenta os 2 grupos (GE1 e GE2), o questionário de Qualidade de Vida para Asma Pediátrica (PedsQL), os elementos da intervenção e sua duração.

\section{Atividades Desenvolvidas pelos grupos experimental 1 e 2}

a) Tratamento manipulativo osteopático (TMO): É um protocolo de técnicas que foi desenvolvido para este estudo objetivando melhorar a função da caixa torácica e respiratória ${ }^{37}$ e por consequência o fluxo linfático, a circulação e as defesas imunológicas dos indivíduos ${ }^{38-40}$. As manobras para a composição do TMO foram utilizadas a partir de $\operatorname{QueF}^{36} \mathrm{e}$ Barral e Mercier ${ }^{41}$. O protocolo constituiu-se de 5 manobras osteopáticas: manipulação combinada do 
centro frênico ${ }^{41}$, estiramento da pleura parietal ${ }^{36,41}$, estiramento do ligamento suspensório da cúpula pleural $^{41}$, mobilização das fáscias endotorácicas ${ }^{36} \mathrm{e}$ equilibração das tensões das fáscias mediastinais ${ }^{36}$.

\section{Manipulaçáo combinada do centro frênico ${ }^{41}$}

A técnica de manipulação combinada do centro frênico realiza-se com o participante do estudo sentado na mesa de tratamento numa posição cifosada. O terapeuta encontra-se em pé atrás do participante, colocando o bordo ulnar das mãos abaixo do gradil costal mantendo uma pressão suave e firme contra o diafragma. O terapeuta mobiliza o tórax do participante até achar uma posição de relaxamento do diafragma sentido nas próprias mãos. A técnica é concluída quando o diafragma relaxa.

\section{Estiramento da pleura parietal ${ }^{36,41}$}

O estiramento da pleura parietal foi adaptado para a posição do participante em decúbito dorsal e o terapeuta sentado em uma cadeira na cabeceira da mesa de tratamento. Foram estiradas as pleuras da regiáo superior do tórax de ambos os lados. Para realizar o estiramento da pleura parietal do lado direito, solicita-se que o participante do estudo coloque a mão direita atrás da cabeça, depois o terapeuta move a junção crânio-cervical superiormente e rodava para a esquerda (liberando as inserçōes posteriores da pleura) ou para a direita (para as inserções anteriores) e ao mesmo tempo pressionavam-se as costelas do lado esquerdo inclinando o tórax para a esquerda salientando o lado direito.

\section{Estiramento do ligamento suspensório da cúpula pleural ${ }^{41}$}

O estiramento do ligamento suspensório da cúpula pleural foi adaptado para a posição do participante do estudo em decúbito dorsal e o terapeuta sentado em uma cadeira na cabeceira da mesa de tratamento. Para estirar o ligamento, foi necessário mover a cabeça e a cervical no sentido oposto ao lado do tórax que seria tratado. Neste caso tratou-se os dois lados. O efeito do estiramento foi acentuado pela adição de uma inclinação lateral da cabeça, e uma depressão inferior e medial da porção superior do tórax no lado a ser tratado. A manobra foi ritmicamente feita até que ocorresse uma liberação tecidual, ou seja, um relaxamento.

\section{Mobilizaçáo das fáscias endotorácicas ${ }^{36}$}

A mobilização das fáscias endotorácicas foi adaptada para a posição do indivíduo em decúbito dorsal estando o terapeuta sentado em uma cadeira na cabeceira da mesa de tratamento. A técnica para ser realizada em todo o pulmáo, necessitou fazer uma divisão do tórax em três partes: região superior, médio e inferior. O terapeuta manteve uma pressão com as mãos em uma das regióes bilateralmente durante o período de expiração e aliviava bruscamente em uma inspiração, sendo realizadas duas vezes em cada regiáo.

\section{Equilibração das tensóes das fáscias mediastinais $^{36}$}

A equilibração das tensões das fáscias mediastinais foi realizada com o participante do estudo em decúbito dorsal e o terapeuta sentado em uma cadeira na cabeceira da mesa de tratamento. $\mathrm{O}$ terapeuta segura com a mão cefálica a região occiptal do indivíduo, mantendo a polpa dos dedos sob a linha curva occiptal. Apoia a mão suavemente no esterno do participante, seguindo o eixo da $3^{\text {a }}$ à $6^{a}$ cartilagem costal. Após, ocorre um aumento no apoio do esterno, tracionando a curva occiptal na sua direção.

b) Exercícios aquáticos: Foram realizados através da natação com a técnica dos nados crawl e costas. Desenvolveu-se após perceber que o grupo estava com domínio e segurança no meio-líquido. Esta foi através de uma estruturação de prática parcialprogressiva com exercícios de flutuação, deslize, movimentação técnica de pernas, braços e respiração dos nados crawl e costas. Após esta sequência de educativos e corretivos, que perdurou por 8 sessóes, priorizou-se as execuçóes com distância progressivas e intensidade em $75 \%$ da condição de cada executante. A intensidade foi calculada pela frequência de pulso (FP), individualmente, em relaçáo à idade e ao meio aquático, utilizandose a fórmula $\left[\mathrm{FCM}=(220-\right.$ idade $)-12 \mathrm{bpm}^{42}$.

c) Ginástica respiratória: desenvolveu-se através do relaxamento corporal através do Método Jacobson, onde se realizava contração e relaxamento de 3 a 5 segundos, parcializando os segmentos corporais a partir de orientaçóes de RissARDI E GoDoY $^{18}$. Após, ocorreu a respiração diafragmática ensinada aos praticantes progressivamente na posição deitada, sentada, e em pé ${ }^{17}$. 


\section{Análise estatística}

Realizou-se análise descritiva dos dados e após verificou-se a distribuição destes através do teste de Shapiro-Wilk. Comprovada a normalidade dos dados para ambos os grupos e dimensóes, utilizou-se o teste $\mathrm{T}$ de Student para amostras pareadas para verificar a diferença entre pré e pós intervenção. Foi utilizado ainda, o teste para amostras independentes para identificar a diferença entre os dois grupos no pós-teste. Usou-se o aplicativo SPSS for Windows, versão 21.0 com nível de significância de 5\%.

\section{Resultados}

Após o desenvolvimento das atividades nos dois grupos experimentais (GE1 e GE2), percebeuse efeitos positivos em ambos, porém no GE1 os benefícios foram mais acentuados, pois ao aplicarmos o questionário de Qualidade de Vida para Asma Pediátrica (PedsQL ${ }^{\mathrm{TM}}$ ) obteve-se como resultados a melhoria da qualidade de vida em todos seus apectos (físico, emocional, social e escolar). Isto foi comprovada através da diferença estatisticamente significativa entre pré e pós intervenção (TABELA 2) e através da comparação também entre os pós testes nos dois grupos (GE1 e GE2), conforme TABELA 4.

O Grupo Experimental 2 (GE2) beneficiou-se da melhoria da qualidade de vida, mas somente no aspecto social, nos demais funcionamentos (físico, emocional e escolar) esta diferença não foi comprovada estatisticamente, apesar das médias apontarem uma visível diminuição, o que indica melhorias, conforme TABELA 3.

TABELA 2 - Comparação das dimensões no Questionário de Qualidade de Vida do Grupo Experimental 1 (11 sujeitos), entre Pré e Pós-Teste

\begin{tabular}{lcccc}
\hline $\begin{array}{l}\text { Qualidade de vida }- \\
\text { dimensóes }\end{array}$ & $\begin{array}{c}\text { Pré-teste } \\
\text { (média } \pm \text { desvio padráo) }\end{array}$ & $\begin{array}{c}\text { Pós-teste } \\
\text { (média } \pm \text { desvio padráo) }\end{array}$ & T & P \\
\hline Física & $28,36 \pm 2,01$ & $12,91 \pm 1,97$ & 15,40 & $<0,001^{*}$ \\
Emocional & $19,18 \pm 0,75$ & $10,64 \pm 1,43$ & 14,40 & $0,002^{*}$ \\
Social & $19,09 \pm 1,04$ & $8,45 \pm 1,29$ & 18,98 & $<0,001^{*}$ \\
Escolar & $18,91 \pm 1,04$ & $8,55 \pm 1,21$ & 13,12 & $<0,001^{*}$ \\
\hline
\end{tabular}

TABELA 3 - Comparação das dimensões no Questionário de Qualidade de Vida do Grupo Experimental 2 (7 sujeitos), entre Pré e Pós-Teste

\begin{tabular}{lcccc}
\hline $\begin{array}{l}\text { Qualidade de vida }- \\
\text { dimensóes }\end{array}$ & $\begin{array}{c}\text { Pré-teste } \\
\text { (média } \pm \text { desvio padráo) }\end{array}$ & $\begin{array}{c}\text { Pós-teste } \\
\text { (média } \pm \text { desvio padráo) }\end{array}$ & T & p \\
\hline Física & $27,71 \pm 1,11$ & $18,80 \pm 1,45$ & $-3,80$ & 0,642 \\
Emocional & $16,09 \pm 1,84$ & $15,57 \pm 2,16$ & $-3,39$ & 0,747 \\
Social & $17,71 \pm 1,13$ & $14,43 \pm 1,34$ & 3,57 & $0,012^{*}$ \\
Escolar & $12,72 \pm 1,60$ & $11,19 \pm 1,97$ & 1,22 & 0,129 \\
\hline
\end{tabular}

Ao utilizarmos o teste $t$ para amostras independentes com os dados do pós-teste do GE1 e do pós-teste do GE2 obtivemos diferença estatisticamente significativa nos funcionamentos físico, emocional, social e escolar (TABELA 4).
Demonstrando com isto a superioridade do grupo que praticou os exercícios combinados de TMO, exercícios aquáticos e a ginástica respiratória em relação ao grupo que só praticou exercícios aquáticos e a ginástica respiratória. 
TABELA 4 - Comparação das dimensões no Questionário de Qualidade de Vida, entre Grupo Experimental 1 (11) e Grupo Experimental 2 (7 sujeitos) no pós-teste

* Diferença estaticamente significativa, com $p \leq 0,05$.

\begin{tabular}{lcccc}
\hline $\begin{array}{l}\text { Qualidade de vida }- \\
\text { dimensóes }\end{array}$ & $\begin{array}{c}\text { Pós-teste GE1 } \\
\text { (média } \pm \text { desvio padráo) }\end{array}$ & $\begin{array}{c}\text { Pós-teste GE2 } \\
\text { (média } \pm \text { desvio padráo) }\end{array}$ & T & P \\
\hline Física & $12,91 \pm 1,97$ & $18,80 \pm 1,45$ & 3,81 & $0,02^{*}$ \\
Emocional & $10,647 \pm 1,43$ & $15,57 \pm 2,16$ & 2,19 & $<0,01^{*}$ \\
Social & $8,45 \pm 1,29$ & $14,43 \pm 1,34$ & 3,17 & $<0,01^{*}$ \\
Escolar & $8,55 \pm 1,21$ & $11,19 \pm 1,97$ & 1,99 & $<0,01^{*}$ \\
\hline
\end{tabular}

\section{Discussão}

Neste trabalho foram aplicados dois programas interventivos: no GE1 foi combinado a TMO, Exercícios Aquáticos e Ginástica Respiratória e no GE2 somente os Exercícios Aquáticos e Ginástica Respiratória. Este último conforme a literatura ${ }^{13,14-16,20}$ representa práticas já consagradas em relação aos seus efeitos benéficos no tratamento de crianças e adolescentes asmáticos e neste estudo, ao analisarmos a qualidade de vida, obteve-se somente melhorias estatisticamente significativas no aspecto social. Entretanto, teve-se no GE2, que utilizou também o TMO, ganhos mais expressivos e comprovados estatisticamente, nas dimensóes relacionadas à qualidade de vida.

Priorizando benefícios de ordem física, emocional, social e escolar; encontramos no estudo de Bockenhauer et al. ${ }^{24}$, dez pacientes acima dos 18 anos asmáticos crônicos que nunca haviam sido submetidos à TMO. O método foi de pré-teste e pós-teste cruzado, onde os próprios testados serviram de controle por placebo. Cinco desses, na primeira semana foram tratados com manipulaçóes osteopáticas e na segunda semana tratados com placebo, e os outros 5 receberam o placebo primeiro e depois o TMO. Foram avaliados 15 minutos antes e após cada intervenção a excursão torácica em dois locais por uma fita métrica, obtidos também medições de pico de fluxo expiratório. O resultado demonstrou que todos se sentiram relaxados após os procedimentos de TMO e placebo, com aprimoramento significativo do aumento da excursão respiratória tanto na parte superior da caixa torácica quanto na parte inferior após o TMO mais do que no placebo, entretanto o pico de fluxo expiratório não obteve variação significativa. $\mathrm{O}$ aumento da excursão da caixa torácica pode ser uma das explicaçóes para a melhora respiratória dos pacientes asmáticos do GE que realizaram a TMO, melhorando a qualidade de vida nos aspectos físicos relacionados ao ganho de força, melhor realização das tarefas domésticas, diminuição do cansaço e mais disposição para caminhar e correr.

Em pesquisa de Guiney et al. ${ }^{23}$, foram avaliados o pico de fluxo expiratório em crianças asmáticas de 5 a 17 anos pré e pós a utilização de TMO em 90 sujeitos e pré e pós o placebo em mais 50 sujeitos, obtendo um resultado significativo de melhora da função pulmonar, e esta trouxe, consequentemente, melhorias no aspecto físico e funcional dos pacientes. Estes dados foram comprovados pelo pico de fluxo expiratório após a aplicação da TMO e comparado ao placebo, corroborando com o presente estudo que obteve resultados de melhora da qualidade de vida mais significativamente no GE que continha a TMO.

Em um estudo de revisão de literatura ${ }^{43}$, que teve como um dos objetivos resumir os resultados de estudo controlado e randomizado sobre a eficácia da TMO em pacientes adultos com pneumonia diagnosticada, chegaram a conclusão que a TMO pode melhorar a função da caixa torácica, o fluxo linfático e circulatório e que a melhora se dá pela utilização de técnicas diretas sobre a caixa torácica.

Ao unir os benefícios dos exercícios aquáticos ${ }^{44}$, com os benefícios da ginástica respiratória ${ }^{12}$ e a progressão da função respiratória proporcionada pela $\mathrm{TMO}^{23,36}$ encontramos uma relaçáo direta com a melhoria da qualidade de vida também nas dimensóes emocionais, sociais e escolares. Isto foi comprovado significativamente conforme TABELA 4 e expresso, conforme respostas às questóes no instrumento avaliativo, nos aspectos relacionados à diminuiçáo do medo, melhoria da qualidade do sono, novas possibilidades de 
fazer amizades e manter as já existentes, melhoria das demandas atencionais na escola e capacidade aumentada de aprendizagem.

Com a melhora dos elementos destacadas acima, os participantes do estudo diminuíram a internação em hospitais, liberando espaços nestes locais para outros indivíduos com outras patologias, podendo ainda diminuir custos para os órgãos públicos. A literatura mostra os aspectos relacionados à qualidade de vida, também diminui o absenteísmo na escola, consequentemente ocorre o favorecimento do desempenho escolar, contribuindo para um melhor aprendizado ${ }^{45}$.

Tratando-se especificamente da melhora em elementos relacionados com a qualidade de vida através de exercícios aquáticos têm-se alguns estudos ${ }^{2,10,12,16,46}$ já evidenciando-a através da aquisição de uma mudança na respiração durante o sono, na qualidade da alimentação, no controle da doença, na redução do excesso de peso, melhoria postural, melhoria na disposição dos sujeitos para a realização de brincadeiras com colegas e amigos e inclusive na diminuição do absenteísmo escolar.

Encontrou-se na literatura alguns estudos ${ }^{43,47}$ que não evidenciaram resultados positivos na utilização da manipulação osteopática em indivíduos com sintomas respiratórios. Em estudo de BALON et al. ${ }^{48}$ usando a quiropraxiano tratamento com crianças asmáticas nos níveis leve ou moderado, não obteve melhoras significativas, isto pode ser interpretado pela necessidade da associação com alguma terapia manual ou outra técnica auxiliar, que foi o que aconteceu no presente estudo.

O exercício físico quando prescrito adequadamente, torna-se importante para a qualidade de vida de crianças asmática, isto foi comprovados nos estudos de Silva et al..$^{2}$; Rezende et al. ${ }^{5}$; Brockmann et al. ${ }^{6}$; Cassol et al. ${ }^{8}$; Moisés ${ }^{9}$; Betio, Krebs e Keulen ${ }^{11}$; Moisés ${ }^{15}$; Neder et al. ${ }^{49}$; Costa $^{50}$; van Veldhoven et al. ${ }^{46}$ e BASARAn et al. ${ }^{51}$ através da melhoria das dimensões físicas, mais especificamente com benefícios na flexibilidade, na condição de correr determinadas distâncias, na resistência aeróbica, na capacidade pulmonar e na diminuição da frequência cardíaca após o exercício.

O grupo experimental 2, conforme TABELA 3, melhorou significativamente no aspecto social, isto provavelmente se deu ao fato de que executando atividades aquáticas e a ginástica respiratória em grupo, houve melhoria nos contatos sociais, na condição de fazer amizades novas e favorecimento de desafios em conjunto com crianças e adolescentes de mesma idade.

A literatura ainda carece de investigaçóes sobre os benefícios da osteopatia, no entanto pelos achados deste estudo, acredita-se na uniáo da osteopatia, com técnicas respiratórias em meio terrestre e aquático, em pacientes com problemas respiratórios, pois esta combinaçáo pode ser o diferencial na melhora da função pulmonar com a diminuição das frustrações, da aflição, da irritaçáo, da qualidade do sono, maior possibilidade de acompanhar colegas nas brincadeiras e diminuiçáo do desconforto respiratório, todos objetos de interesse na presente investigação, pois estão relacionados à qualidade de vida.

O TMO foi o diferencial entre os grupos, e este unindo-se aos exercícios aquáticos e à ginástica respiratória bem orientados, causou uma melhora significativamente às dimensóes física, emocional e social e escolar. Acredita-se que este tipo de estudo interdisciplinar, pode ser extremamente benéfico para a sociedade escolar, favorecendo diretamente indivíduos asmáticos e seus familiares e inclusive diminuindo os custos de tratamento clínico e medicamentoso no serviço de saúde pública no Brasil.

Próximos estudos deverão ser realizados testando variáveis mais específicas com a utilização de instrumentos mais quantitativos como o expirômetro e pico de fluxo expiratório entre outros marcadores biológicos para dar maior sustentação aos achados nesta temática.

\section{Abstract}

Benefits of the combination among osteopathic manipulation, aquatic exercise and respiratory gymnastics for quality of life in asthmatics children and teenagers

Developing physical exercise programs to improve the quality of life in asthmatics can positively influence the performance of social and sports activities by this public. The aim of this study was to investigate the effect of the addition of Osteopathic Manipulative Treatment (BMT) to sessions of respiratory exercise exercises and aquatic exercises to improve the quality of life of asthmatics. The study was developed with 
18 participants, aged between 8 and 15 years, of both sexes and students of Elementary School I and II. Eleven (11) were part of the experimental group 1 (EG1) and carried out a program of OMT, aquatic exercises and respiratory gymnastics. Seven (7) individuals were part of the experimental group two (EG2) and received aquatic exercises and respiratory gymnastics. Both were tested before and after completion of 16 sessions of OMT and 32 sessions of others practices, through the Quality of Life Questionnaire for Pediatric Asthma (PedsQL). For data analysis, after proof of normality, we used the Student $t$ test for paired samples and the $T$ test for independent samples, using SPSS, version 21.0 with a significance level of 5\%. The results indicated a statistically significant difference in the social, school, physical and emotional dimensions of the questionnaire in the EG when compared between the beginning and the end of the program and confirmed the statistically significant difference in the post test in both groups, prevailing improvements in all dimensions for the group that had OMT, aquatic exercises and respiratory gymnastics. The results strengthen the idea of the efficacy of the combination of these practices improve the quality of life of these children.

KeYwoRDS: Asthma; Exercise Therapy; Osteopathic Manipulation; Swimming; Breathing Exercises.

\section{Referências}

1. Oliveira MA, Muniz MT, Santos LA, Faresin SM, Fernandes AL. Custo-efetividade de programa de educação para adultos asmáticos atendidos em hospital-escola de instituição pública. J Pneumologia. 2002;28(2):71-6.

2. Silva CS, Torres LAGM, Rahal A, Filho JT, Vianna E. Avaliação de um programa de treinamento físico por quatro meses para crianças asmáticas. J Bras Pneumol. 2005;31(4):279-85.

3. Anandan C, Nurmatov U, van Schayck OC, Sheikh A. Is the prevalence of asthma declining? Systematic review of epidemiological studies. Allergy. 2010;65(2):152-67.

4. Pitanga FJG. Epidemiologia da atividade física, exercício físico e saúde. 2a ed. São Paulo: Phorte; 2004.

5. Rezende IMO, Moura ALD, Costa BC, et al. Efeitos da reabilitação pulmonar sobre a qualidade de vida: uma visão das crianças asmáticas e de seus pais. Acta Fisiátrica. 2006;15(3):165-9.

6. Brockmann VP, Caussade LS, Holmgren PN, et al. Actividad física y obesidad em ninôs com asma. Rev Chil Pediatr. 2007;78(5):482-8.

7. Silva FM, Santos AC, Miranda J. As atividades físicas na recuperação das crianças portadoras de deficiência respiratória. Rev Cent Ciênc Saúde. 1994;13(4):16-21.

8. Cassol VE, Trevisan ME, Moraes EZC, Portela LOC, Barreto SSM. Broncoespasmo induzido pelo exercício em crianças e adolescentes com diagnóstico de asma. Rev Bras Pneumol. 2004;30(2):102-8.

9. Moisés MP. Ginástica respiratória para asmáticos: efeito de redução do número e intensidade de crises asmáticas. Rev Mack Educ Fís Esporte. 2006;5(especial):73-81.

10. Moraes GML, Novo NF, Juliano Y, Cury MCFS, Bogossian M. Comportamento do fluxo expiratório antes e após aula de natação em crianças portadoras de asma. Rev Soc Bras Clín Med. 2007;5(1):7-13.

11. Betio J, Krebs RJ, Keulen GEV. Atividade física para portadores de asma. Cinergis. 2007;8(2):7-12.

12. Antes DL, Katzer J, Lorensi C, Santos R, Contreira A, Corazza S. Aspectos gerais da saúde em portadores de enfermidades respiratórias praticantes de um programa de atividades físicas regulares. Lect Educ Fís Deportes [Internet]. 2008 [citado 30 jul 2019];13(123). Disponível em: https://www.efdeportes.com/efd123/portadores-de-enfermidades-respiratorias-praticantesde-atividades-fisicas-regulares.htm.

13. Silva MCR, Corazza ST, Katzer JI, Mota CB, Soares JC. Equilíbrio corporal em crianças asmáticas e não asmáticas. Motriz. 2013;19(2):480-6.

14. Cabral AL, Teixeira LR, editores. Vencendo a asma: uma abordagem multidisciplinar. São Paulo: Bevilacqua; 1994.

15. Moisés MP, organizadora. Atividades físicas e a criança asmática. Brasília: Ministério da Educação e do Desporto; 1993.

16. Weisgerber MC, Guill M, Weisgerber JM, Butler H. Benefits of swimming in asthma: effect of a session of swimming lessons on symptoms and PFTs with review of the literature. J Asthma. 2003;40(5):453-64.

17. Corazza ST, Silva MCR, Paulus LD, Trindade CRP, Vidor DM. Asma infantil - esclarecimentos e uma proposta de intervenção motora, física e funcional. Pensar Prát. 2016;19(1):232-44.

262 • Rev Bras Educ Fís Esporte, (São Paulo) 2019 Abr-Jun;33(2):255-64 
18. Rissardi GGL, Godoy MF. Estudo da aplicação da técnica de relaxamento muscular progressivo de Jacobson modificada nas respostas das variáveis cardiovasculares e respiratórias de pacientes hansenianos. Arq Ciênc Saúde. 2007;14(3):175-80.

19. Souza Filho PG. Introdução aos métodos de relaxamento. 140 Congresso Brasileiro de Psicoterapias Corporais; 1-3 maio 2009; Curitiba, BR. Curitiba: Centro Reichiano; 2009.

20. Contreira AR, Salles SN, Silva MP, Antes DL, Katzer JI, Corazza ST. O efeito da prática regular de exercícios físicos no estilo de vida e desempenho motor de crianças e adolescentes asmáticos. Pensar Prát. 2010;13(1):1-16.

21. Allen TW, D’Alonzo GE. Investigating the role of osteopathic manipulation in the treatment of asthma. J Am Osteopath Assoc. 1993;93(6):654-6, 659.

22. Noll DR, Degenhardt BF, Johnson JC, Burt SA. Immediate effects of osteopathic manipulative treatment in elderly patients with chronic obstructive pulmonary disease. J Am Osteopath Assoc. 2008;108(5):251-9.

23. Guiney PA, Chou R, Vianna A, Lovenheim J. Effects of osteopathic manipulative treatment on pediatric patients with asthma: a randomized controlled trial. J Am Osteopath Assoc. 2005;105(1):7-12.

24. Bockenhauer SE, Julliard KN, Lo KS, Huangs E, Sheth AM. Quantifiable effects of osteopathic manipulative techniques on patients with chronic asthma. J Am Osteopath Assoc. 2002;102(7):371-5.

25. Spaeth DG, Pheley AM. Use of osteopathic manipulative treatment by Ohio osteopathic physicians in various specialties. J Am Osteopath Assoc. 2003;103(1):16-26.

26. Schwerla F, Kaiser AK, Gietz R, Kastner R. Osteopathic treatment of patients with long-term sequelae of whiplash injury: effect on neck pain disability and quality of life. J Altern Complement Med. 2013;19(6):543-9.

27. Diniz LR, Nesi J, Curi AC, Martins W. Qualitative evaluation of osteopathic manipulative therapy in a patient with gastroesophageal reflux disease: a brief report. J Am Osteopath Assoc. 2014;114(3):180-8.

28. Hubert D, Soubeiran L, Gourmelon F, et al. Impact of osteopathic treatment on pain in adult patients with cystic fibrosis - a pilot randomized controlled study. Plos One. 2014;9(7):e102465.

29. Licciardone JC, Buchanan S, Hensel KL, King HH, Fulda KG, Stoll ST. Osteopathic manipulative treatment of back pain and related symptoms during pregnancy: a randomized controlled trial. Am J Obstet Gynecol. 2010;202(1):43.e1-8.

30. Apfelbacher C, Paudyal P, Bülbül A, Smith H. Measurement properties of asthma-specific quality-of-life measures: protocol for a systematic review. Syst Rev. 2014;3:83.

31. Luna-Pech JA, Torres-Mendoza BM, Luna-Pech JA, Garcia-Cobas CY, Navarrete-Navarro S, Elizalde-Lozano AM. Normocaloric diet improves asthma-related quality of life in obese pubertal adolescents. Int Arch Allergy Immunol. 2014;163(4):252-8.

32. Thomas JR, Nelson JK, Silverman SJ. Métodos de pesquisa em atividade física. 5a ed. Porto Alegre: Artmed; 2007.

33. Sociedade Brasileira de Pneumologia e Tisiologia, Sociedade Brasileira de Alergia e Imunopatologia, Sociedade Brasileira de Pediatria, Sociedade Brasileira de Clínica Médica. III Consenso Brasileiro no Manejo da Asma. J Pneumol. 2002;28(Supl 1):S4-28.

34. Varni JW, Burwinkle TM, Rapoff MA, Kamps JL, Olson N. The Peds QL in pediatric asthma: reability and validity of the Pediatric Quality of Life Inventory generic core scales and asthma module. J Behav Med. 2004;27(3);297-318.

35. Roncada C, Mattiello R, Pitrez P, Sarria EE. Instrumentos específicos para avaliar a qualidade de vida em crianças e adolescentes asmáticos. J Pediatr (Rio J). 2013;89(3):3-13.

36. Quef BH. Técnicas osteopáticas viscerais. São Paulo: Santos; 2008.

37. Yao S, Hassani J, Gagne M, George G, Gilliar W. Osteopathic manipulative treatment as a useful adjunctive tool for pneumonia. J Vis Exp. 2014;6(87). doi: 10.3791/50687.

38. Saggio G, Docimo S, Pilc J, Norton J, Gilliar W. Impact of osteopathic manipulative tratamento n secretory immunoglobulin a levels in a stressed population. J Am Osteopath Assoc. 2011;111(3):143-7.

39. Chikly BJ. Manual techniques addressing the lymphatic system: origins and development. J Am Osteopath Assoc. 2005;105(10):457-64.

40. Knott EM, Tune JD, Stoll ST, Downey HF. Increased Lymphatic Flow in the Thoracic Duct During Manipulative Intervention. J Am Osteopath Assoc. 2005;105(10):447-56.

41. Barral JP, Mercier P. Manipulação Visceral I. Teresópolis: Upledger Brasil; 2014.

42. DiCarlo LJ, Sparling PB, Millard-Stafford ML, Rupp JC. Peak heart rate during maximal running and swimming: implications for exercise prescription. Int J Sports Med. 1991;12(3):309-12.

43. Posadzki P, Lee MS, Ernst E. Osteopathic manipulative treatment for pediatric conditions: a systematic review. Pediatrics. 2013;132(1):140-52.

44. Gualdi FR, Tumelero S. Asma e os benefícios da atividade física. Lect Educ Fís Deportes [Internet]. 2004 [citado 30 jul 2019];10(72). Disponível: https://www.efdeportes.com/efd72/asma.htm. 
45. Payrovee Z, Kashaninia Z, Alireza Mahdaviani S, Rezasoltani P. Effect of family empowerment on the quality of life of schoolaged children with asthma. Tanaffos. 2014;13(1):35-42.

46. Van Veldhoven NH, Vermeer A, Bogaard JM, et al. Children with asthma and physical exercise: effects of an exercise programme. Clin Rehabil. 2001;15(4):360-70.

47. Hondras MA, Linde K, Jones AP. Manual therapy for asthma. Cochrane Database Syst Rev. 2005;18(2):CD001002.

48. Balon J, Aker PD, Crowther ER, et al. A comparison of active and simulated chiropractic manipulation as adjunctive treatment or childhood asthma. N Engl J Med. 1998;339(15):1013-20.

49. Neder JA, Nery LE, Silva AC, Cabral ALB, Fernandes ALG. Short term effects of aerobic training in the clinical management of moderate to severe asthma in children. Thorax. 1999;54(3):202-6.

50. Costa NP. Resultados de um programa de tratamento com ou sem treinamento físico em crianças com asma [tese]. São Paulo (SP): Universidade Federal de São Carlos, Escola de Medicina; 2001.

51. Basaran S, Guler-Uysal F, Ergen N, Seydaoglu G, Bingol-Karakoç G, Ufuk Altintas D. Effects of physical exercise on quality of life, exercise capacity and pulmonary function in childern with asthma. J Rehabil Med. 2006;38(2):130-5.

\begin{tabular}{|c|c|}
\hline $\begin{array}{r}\text { ENDEREÇO } \\
\text { Rafael Cunha Laux }\end{array}$ & Recebido para publicação: 22/05/2015 \\
\hline Avenida Roraima, $1000-$ & 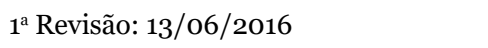 \\
\hline prédio 51, sala 1.021 - Camobi - 97105-900 & 2a Revisão: 28/o8/2017 \\
\hline Santa Maria - RS - BRASIL & 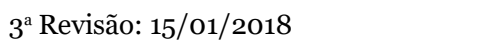 \\
\hline $\begin{array}{l}\text { e-mail: rafael-laux@hotmail.com; } \\
\text { aprendizagem.motoraufsm@gmail.com }\end{array}$ & Aceito: 07/03/2018 \\
\hline
\end{tabular}

